\title{
The number of $k$-parallelogram polyominoes
}

\author{
Daniela Battaglino $^{1,2} \quad$ Jean Marc Fedou ${ }^{2} \quad$ Simone Rinaldi $\|^{\ddagger} \quad$ Samanta Socci ${ }^{1}$ \\ ${ }^{1}$ Dipartimento di Matematica e Informatica, Università di Siena, Siena, Italy \\ ${ }^{2}$ Departement d'Informatique, UNS Nice, France
}

\begin{abstract}
A convex polyomino is $k$-convex if every pair of its cells can be connected by means of a monotone path, internal to the polyomino, and having at most $k$ changes of direction. The number $k$-convex polyominoes of given semi-perimeter has been determined only for small values of $k$, precisely $k=1,2$. In this paper we consider the problem of enumerating a subclass of $k$-convex polyominoes, precisely the $k$-convex parallelogram polyominoes (briefly, $k$-parallelogram polyominoes). For each $k \geq 1$, we give a recursive decomposition for the class of $k$ parallelogram polyominoes, and then use it to obtain the generating function of the class, which turns out to be a rational function. We are then able to express such a generating function in terms of the Fibonacci polynomials.
\end{abstract}

Résumé. Un polyomino convexe est dit $k$-convexe lorsqu'on peut relier tout couple de cellules par un chemin monotone ayant au plus $k$ changements de direction. Le nombre de polyominos $k$-convexes n'est connu que pour les petites valeurs de $k=1,2$. Dans cet article, nous énumérons la sous classes des polyominos $k$-convexes qui sont également parallélogramme, que nous appelons $k$-parallelogrammes. Nous donnons une décomposition récursive de la classe des polyominos $k$-parallélogrammes pour chaque $k$, et en déduisons la fonction génératrice, rationnelle, selon le demi-périmètre. Nous donnons enfin une expression de cette fonction génératrice en terme des polynômes de Fibonacci.

Keywords: Convex polyominoes, L-convex polyominoes, Rational generating functions, Fibonacci polynomials

\section{Introduction}

In the plane $\mathbb{Z} \times \mathbb{Z}$ a cell is a unit square and a polyomino is a finite connected union of cells having no cut point. Polyominoes are defined up to translations. A column (row) of a polyomino is the intersection between the polyomino and an infinite strip of cells whose centers lie on a vertical (horizontal) line. A polyomino is said to be column-convex (row-convex) when its intersection with any vertical (horizontal) line is convex. A polyomino is convex if it is both column and row convex (see Figure $1(a)$ ). In a convex polyomino the semi-perimeter is given by the sum of the number of rows and columns, while the area is the number of its cells. For more definitions on polyominoes, we address the reader to [1].

The number $f_{n}$ of convex polyominoes with semi-perimeter $n \geq 2$ was obtained by Delest and Viennot in [7], and it is:

$$
f_{n+2}=(2 n+11) 4^{n}-4(2 n+1)\left(\begin{array}{c}
2 n \\
n
\end{array}\right), \quad n \geq 0 ; \quad f_{0}=1, \quad f_{1}=2 .
$$

\footnotetext{
†Supported by PRIN Project 2011: “Automi e Linguaggi Formali: Aspetti Matematici e Applicativi” 
The study of this paper originates from the work on $k$-convex polyominoes by Castiglione and Restivo in [5]. Their idea is to consider paths internal to polyominoes, where a path is simply a self-avoiding sequence of unit steps of four types: north $n=(0,1)$, south $s=(0,-1)$, east $e=(1,0)$, and west $w=(-1,0)$, entirely contained in the polyomino. A path connecting two distinct cells, $A$ and $B$, starts from the center of $A$, and ends at the center of $B$ (see Figure $1(b)$ ). We say that a path is monotone if it is constituted only of steps of two types (see Figure $1(c)$ ). Given a path $w=u_{1} \ldots u_{k}$, each pair of steps $u_{i} u_{i+1}$ such that $u_{i} \neq u_{i+1}, 0<i<k$, is called a change of direction.

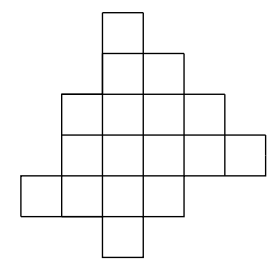

(a)

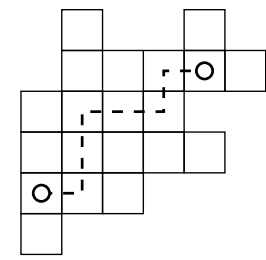

(b)

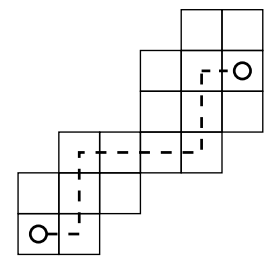

(c)

Figure 1: $(a)$ a convex polyomino; $(b)$ a monotone path between two cells of the polyomino with four changes of direction; (c) a 4-parallelogram (non 3-parallelogram) polyomino.

In [5] the authors observed that convex polyominoes have the property that every pair of cells is connected by a monotone path, and proposed a classification of convex polyominoes based on the number of changes of direction in the paths connecting any two cells of a polyomino. More precisely, a convex polyomino is $k$-convex if every pair of its cells can be connected by a monotone path with at most $k$ changes of direction, and $k$ is called the convexity degree of the polyomino.

For $k=1$ we have the $L$-convex polyominoes, where any two cells can be connected by a path with at most one change of direction. In recent literature $L$-convex polyominoes have been considered from several points of view: in [5] it is shown that they are a well-ordering according to the sub-picture order; in [2] the authors have investigated some tomographical aspects, and have discovered that $L$-convex polyominoes are uniquely determined by their horizontal and vertical projections. Finally, in [3, 4] it is proved that the number $l_{n}$ of $L$-convex polyominoes having semi-perimeter equal to $n+2$ satisfies the recurrence relation

$$
l_{n+2}=4 l_{n+1}-2 l_{n}, \quad n \geq 1, \quad l_{0}=1, \quad l_{1}=2 \quad l_{2}=7 .
$$

For $k=2$ we have 2-convex (or $Z$-convex) polyominoes, such that each two cells can be connected by a path with at most two changes of direction. Unfortunately, $Z$-convex polyominoes do not inherit most of the combinatorial properties of $L$-convex polyominoes. In particular, their enumeration resisted standard enumeration techniques and it was obtained in [8] by applying the so-called inflation method. The authors proved that the generating function is algebraic and that the sequence asymptotically grows as $n 4^{n}$, that is the same growth of the whole class of the convex polyominoes.

However, the solution found for 2-convex polyominoes seems to be not easily generalizable to a generic $k$, hence the problem of enumerating $k$-convex polyominoes for $k>2$ is still open and difficult to solve. Recently, some efforts in the study of the asymptotic behavior of $k$-convex polyominoes have been made by Micheli and Rossin in [9]. 
In order to probe further, in this paper we tackle the problem of enumerating a remarkable subclass of $k$-convex polyominoes, precisely the $k$-convex polyominoes which are also parallelogram polyominoes, called for brevity $k$-parallelogram polyominoes.

We recall that a parallelogram polyomino is a polyomino whose boundary can be decomposed in two paths, the upper and the lower paths, which are made of north and east unit step and meet only at their starting and final points. Figure 1 (c) depicts a 4-parallelogram (non 3-convex) polyomino.

Moreover, it is well known [11] that the number of parallelogram polyominoes with semi-perimeter $n \geq 2$ is equal to the $(n-1)$ th Catalan number, where Catalan numbers are defined by

$$
c_{n}=\frac{1}{n+1}\left(\begin{array}{c}
2 n \\
n
\end{array}\right) .
$$

The class of $k$-parallelogram polyominoes can be treated in a simpler way than $k$-convex polyominoes, since we can use the simple fact that a parallelogram polyomino is $k$-convex if and only if every cell can be reached from the lower leftmost cell by at least one monotone path having at most $k$-changes of direction.

Using such a property, we will be able to enumerate $k$-parallelogram polyominoes according to the semi-perimeter, for all $k$. More precisely, in the next sections we will partition the class of $k$-parallelogram polyominoes into three subclasses, namely the flat, right, and up $k$-parallelogram polyominoes. We will provide an unambiguous decomposition for each of the three classes, so we will use these decompositions in order to obtain the generating function of the three classes and then of $k$-parallelogram polyominoes. An interesting fact is that, while the generating function of parallelogram polyominoes is algebraic, for every $k$ the generating function of $k$-parallelogram polyominoes is rational. Moreover, we will be able to express such generating function in terms of the known Fibonacci polynomials [6].

To our opinion, this work is a first step towards the enumeration of $k$-convex polyominoes, since it is possible to apply our decomposition strategy to some larger classes of $k$-convex polyominoes (such as, for instance, directed $k$-convex polyominoes).

\section{Classification and decomposition of $k$-parallelogram polyomi- noes}

In this section we present some basic definitions which will be useful for the rest of the paper, we provide a classification of $k$-parallelogram polyominoes, and then an unique decomposition for each class.

We start noting that to find out the convexity degree of a parallelogram polyomino $P$ it is sufficient to check the changes of direction required to any path running from the lowest leftmost cell (denoted by $S$ ) to the upper rightmost cell (denoted by $E$ ).

In order to use such a property, we define the vertical (horizontal) path $v(P)$ (respectively $h(P)$ ) as the path - if it exists - internal to $P$, running from $S$, and starting with a north step $n$ (respectively $e$ ), where every side has maximal length (see Figure 2). In the graphical representation, we use a dashed line to represent $v(P)$, and a solid line to represent $h(P)$. We remark that our definition does not work if the first column (resp. row) of $P$ is made of one cell, and then in this case we set by definition that $v(P)$ and $h(P)$ coincide (Figure $2(d)$ ). 
Henceforth, if there are no ambiguities we will write $v$ (resp. $h$ ) in place of $v(P)$ (resp. $h(P)$ ). As the reader can easily check the numbers of changes of direction that $h$ and $v$ require to run from $S$ to $E$ may differ at most by one. The following property is straightforward.

Proposition 1 A polyomino $P$ is $k$-parallelogram if and only if at least one among $v(P)$ and $h(P)$ has at most $k$ changes of direction.

In our study we will deal with the class $\mathbb{P}_{k}$ of $k$-parallelogram polyominoes where the convexity degree is exactly equal to $k \geq 0$, then enumeration of $k$-parallelogram polyominoes will readily follow. According to our definition, $\mathbb{P}_{0}$ is made of horizontal and vertical bars of any length. We further notice that, in the given parallelogram polyomino $P$, there may exist a cell starting from which the two paths $h$ and $v$ are superimposed (see Figure 2(b), (c)). In this case, we denote such a cell by $C(P)$ (briefly, $C$ ). Clearly $C$ may even coincide with $S$ (see Figure 2(d)). If such cell does not exist, we assume that $C$ coincides with $E$ (see Figure $2(\mathrm{a})$ ).

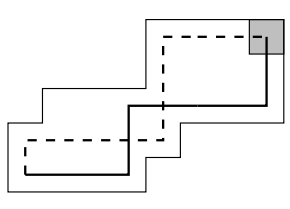

(a)

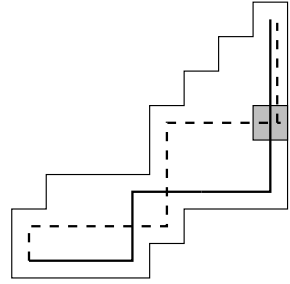

(b)

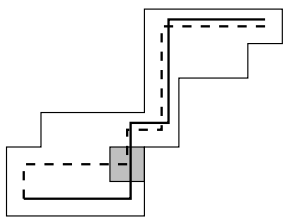

(c)

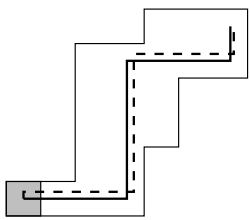

(d)

Figure 2: The paths $h$ (solid line) and $v$ (dashed line) in a parallelogram polyomino, where the cell $C$ has been highlighted (a) A polyomino in $\overline{\mathbb{P}}_{3} ;(b)$ A polyomino in $\mathbb{P}_{3}^{U} ;(c)$ A polyomino in $\mathbb{P}_{4}^{R} ;(d)$ A polyomino in $\mathbb{P}_{3}^{U}$ where $C$ coincides with $S$.

From now on, unless otherwise specified, we will always assume that $k \geq 1$. Let us give a classification of the polyominoes in $\mathbb{P}_{k}$, based on the position of the cell $C$ inside the polyomino:

1. A polyomino $P$ in $\mathbb{P}_{k}$ is said to be a flat $k$-parallelogram polyomino if $C(P)$ coincides with $E$ (see Figure $2(a)$ ). The class of these polyominoes will be denoted by $\overline{\mathbb{P}}_{k}$. According to this definition all rectangles having width and height greater than one belong to $\overline{\mathbb{P}}_{1}$.

2. A polyomino $P$ in $\mathbb{P}_{k}$ is said to be up (resp. right) $k$-parallelogram polyomino, if the cell $C(P)$ is distinct from $E$ and $h$ and $v$ end with a north (resp. east) step. The class of up (resp. right) $k$-parallelogram polyominoes will be denoted by $\mathbb{P}_{k}^{U}\left(\right.$ resp. $\left.\mathbb{P}_{k}^{R}\right)$. Figures $2(b),(c)$, and $(d)$ depict polyominoes in $\mathbb{P}_{k}^{U}$ (resp. $\left.\mathbb{P}_{k}^{R}\right)$.

The reader can easily check that up (resp. right) $k$-parallelogram polyominoes where the cell $C(P)$ is distinct from $S$ can be characterized as those parallelogram polyominoes where $h$ (resp. $v$ ) has $k$ changes of direction and $v$ (resp. $h$ ) has $k+1$ changes of direction.

Now we present a unique decomposition of polyominoes in $\mathbb{P}_{k}$, based on the following idea: given a polyomino $P$, we are able to detect - using the paths $h$ and $v$ - a set of paths on the boundary of $P$, that uniquely identify the polyomino itself. 
More precisely, let $P$ be a polyomino of $\mathbb{P}_{k}$; the path $h$ (resp. $v$ ), when encountering the boundary of $P$, determines $m$ (resp. $m^{\prime}$ ) steps where $m$ (resp. $m^{\prime}$ ) is equal to the number of changes of directions of $h$ (resp. $v$ ) plus one. To refer to these steps we agree that the step encountered by $h$ (resp. $v$ ) for the $i$ th time is called $X_{i}$ or $Y_{i}$ according if it is a horizontal or vertical one (see Fig. 3). We point out that if $P$ is flat all steps $X_{i}$ and $Y_{i}$ are distinct, otherwise there may be some indices $i$ for which $X_{i}=X_{i+1}$ (or $Y_{i}=Y_{i+1}$ ), and this happens precisely with the steps determined after the cell $C(P)$ (see Fig. $4(b),(c)$ ). The case $C(P)=S$ can be viewed as a degenerate case where the initial sequence of north (resp. east) steps of $v$ (resp. $h$ ) has length zero. Therefore the definition of the steps $X_{i}, Y_{i}$ can be given as follows: if the first column (resp. the lowest row) is made of one cell, then we set the step $X_{1}$ (resp. $Y_{1}$ ) to be equal to the leftmost east (resp. lowest north) step of the upper (resp. lower) path of $P$, and all the other steps $X_{i}$ and $Y_{i}$ are determined as before (see Fig. $4(b)$ ).

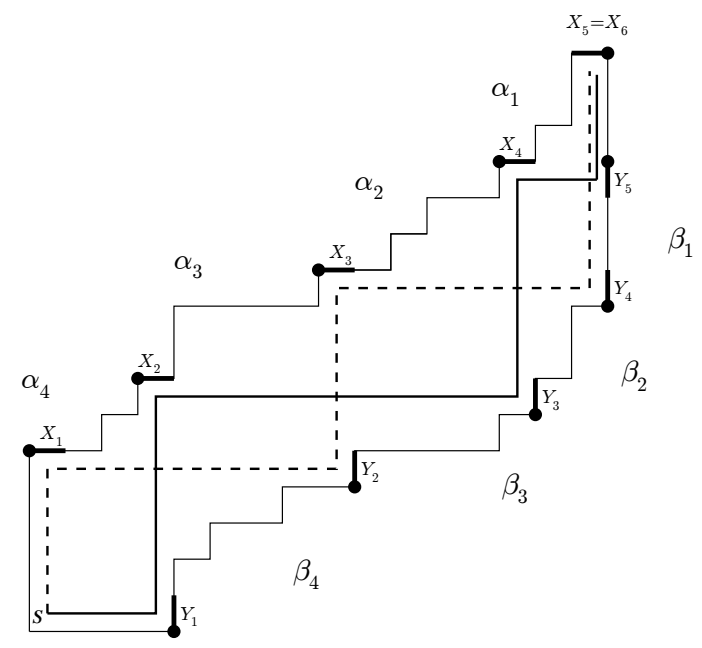

Figure 3: Decomposition of a polyomino of $\mathbb{P}_{4}^{U}$.

Now we decompose the upper (resp. lower) path of $P$ in $k$ (possibly empty) subpaths $\alpha_{1}, \ldots, \alpha_{k}$ (resp. $\beta_{1}, \ldots, \beta_{k}$ ) using the following rule: $\alpha_{1}$ (resp. $\beta_{1}$ ) is the path running from the beginning of $X_{k}$ to end of $X_{k+1}$ (resp. from the beginning of $Y_{k}$ to $Y_{k+1}$ ); let us consider now the $k-1$ (possibly empty) subpaths, $\alpha_{i}$ (resp. $\beta_{i}$ ) from the beginning of $X_{k+1-i}$ (resp. $Y_{k+1-i}$ ) to the beginning of $X_{k+2-i}$ (resp. $Y_{k+2-i}$ ), for $i=2 \cdots k$. We observe that these paths are ordered from the right to the left of $P$. For simplicity we say that a path is flat if it is composed of steps of just one type.

The following proposition provides a characterization of the polyominoes of $\mathbb{P}_{k}$ in term of the paths $\alpha_{1}, \ldots, \alpha_{k}, \beta_{1}, \ldots, \beta_{k}$.

Proposition 2 A polyomino $P$ in $\mathbb{P}_{k}$ is uniquely determined by a sequence of (possibly empty) paths $\alpha_{1}, \ldots, \alpha_{k}, \beta_{1}, \ldots, \beta_{k}$, each of which made by north and east unit steps. Moreover, these paths have to satisfy the following properties:

- $\alpha_{i}$ and $\beta_{i+1}$ must have the same width, for every $i \neq 1$; if $i=1$, we have that $\alpha_{1}$ is always non empty and the width of $\alpha_{1}$ is equal to the width of $\beta_{2}$ plus one; 


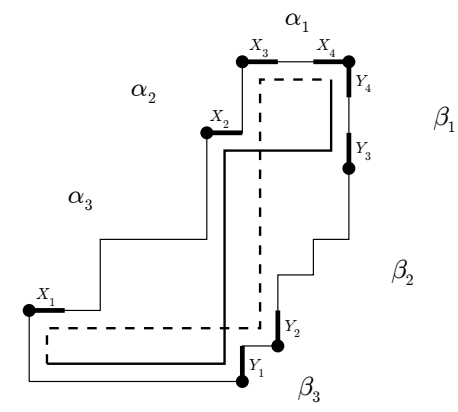

(a)

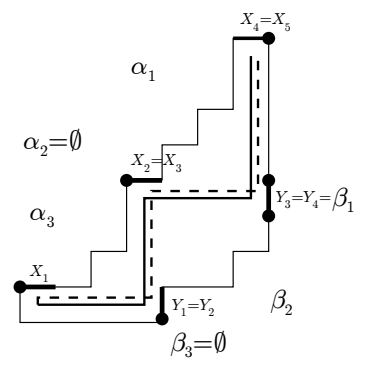

(b)

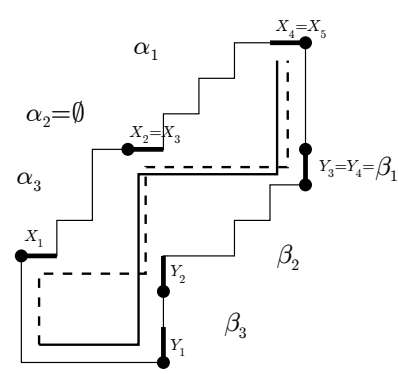

(c)

Figure 4: (a) A polyomino $P \in \overline{\mathbb{P}}_{3}$ in which $\alpha_{1}$ and $\beta_{1}$ are flat and each other path is non empty and non flat. $(b)$ A polyomino $P \in \mathbb{P}_{3}^{U}$ where: $\beta_{3}$ is empty, $\alpha_{2}$ is empty and $\beta_{1}$ is equal to a north unit step. (c) A polyomino $P \in \mathbb{P}_{3}^{U}$ where $\beta_{3}$ is flat, $\alpha_{2}$ is empty and $\beta_{1}$ is equal to a north unit step.

- $\beta_{i}$ and $\alpha_{i+1}$ must have the same height, for every $i \neq 1$; if $i=1$, we have that $\beta_{1}$ is always non empty and the height of $\beta_{1}$ is equal to the width of $\alpha_{2}$ plus one;

- if $\alpha_{i}\left(\beta_{i}\right)$ is non empty then it starts with an east (north) step, $i \geqslant 1$. In particular, for $i=1$, if $\alpha_{1}$ $\left(\beta_{1}\right)$ is different from the east (north) unit step, then it must start and end with an east (north) step.

The semi-perimeter of $P$ is obtained as the sum $\left|\alpha_{1}\right|+\left|\alpha_{2}\right|_{e}+\ldots+\left|\alpha_{k}\right|_{e}+\left|\beta_{1}\right|+\left|\beta_{2}\right|_{n}+\ldots+\left|\beta_{k}\right|_{n}$.

The reader can easily check the decomposition of a polyomino of $\mathbb{P}_{4}^{U}$ in Figure 3 For clarity sake, we need to remark the following consequence of Proposition 2 .

Corollary 1 Let $P \in \mathbb{P}_{k}$ be encoded by the paths $\alpha_{1}, \ldots, \alpha_{k}, \beta_{1}, \ldots, \beta_{k}$. We have:

- for every $i>1$, we have that $\alpha_{i}\left(\beta_{i}\right)$ is empty if and only if $\beta_{i+1}\left(\alpha_{i+1}\right)$ is empty or flat;

- $\alpha_{1}\left(\beta_{1}\right)$ is equal to the east (north) unit step if and only if $\beta_{2}\left(\alpha_{2}\right)$ is empty or flat.

Figure $4(a)$ shows the decomposition of a flat polyomino, $(b)$ shows the case in which $C(P)=S$, so we have that $\beta_{3}$ is empty, then $\alpha_{2}$ is empty, hence $\beta_{1}$ is a unit north step. Figure 4 (c) shows the case in which $h$ and $v$ coincide after the first change of direction and so we have that $\beta_{3}$ is flat, then $\alpha_{2}$ is empty and $\beta_{1}$ is a unit north step.

Now we provide another characterization of the classes of flat, up, and right polyominoes of $\mathbb{P}_{k}$ which directly follows from Corollary 1 and will be used for the enumeration of these objects.

Proposition 3 Let $P$ be a polyomino in $\mathbb{P}_{k}$. We have:

i) $P$ is flat if and only if $\alpha_{1}$ and $\beta_{1}$ are flat and they have length greater than one. It follows that all $\alpha_{i}$ and $\beta_{i}$ are non empty paths, $i=2, \ldots, k$.

ii) $P$ is up (right) if and only if $\beta_{1}\left(\alpha_{1}\right)$ is flat and $\alpha_{1}\left(\beta_{1}\right)$ is non flat. 
The reader can see examples of the statement of Proposition 3 i) in Figure $4(a)$, and of Proposition 3 ii) in Figure $4(b)$ and $(c)$.

As a consequence of Proposition 2 from now on we will encode every polyomino $P \in \mathbb{P}_{k}$ in terms of the two sequences:

$$
\mathcal{A}(P)=\left(\alpha_{1}, \beta_{2}, \alpha_{3}, \ldots, \theta_{k}\right),
$$

with $\theta=\alpha$ if $k$ is odd, otherwise $\theta=\beta$, and

$$
\mathcal{B}(P)=\left(\beta_{1}, \alpha_{2}, \beta_{3}, \ldots, \bar{\theta}_{k}\right),
$$

where $\bar{\theta}=\alpha$ if and only if $\theta=\beta$. The dimension of $\mathcal{A}$ (resp. $\mathcal{B}$ ) is given by $\left|\alpha_{1}\right|+\left|\beta_{2}\right|_{n}+\left|\alpha_{3}\right|_{e}+\ldots$ (resp. $\left|\beta_{1}\right|+\left|\alpha_{2}\right|_{e}+\left|\beta_{3}\right|_{n}+\ldots$ ). In particular, if $C(P)=S$ and $P$ is an up (resp. right) polyomino then $\mathcal{B}(P)=\left(\beta_{1}, \emptyset, \ldots, \emptyset\right)$, (resp. $\left.\mathcal{A}(P)=\left(\alpha_{1}, \emptyset, \ldots, \emptyset\right)\right)$ where $\beta_{1}$ (resp. $\left.\alpha_{1}\right)$ is the north (resp. east) unit step.

\section{Enumeration of $k$-parallelogram polyominoes}

This section is organized as follows: first, we furnish a method to pass from the generating function of the class $\mathbb{P}_{k}$ to the generating function of $\mathbb{P}_{k+1}, k>1$. Then, we provide the enumeration of the trivial cases, i.e. $k=0,1$, and finally apply the inductive step to determine the generating function of $\mathbb{P}_{k}$. The enumeration of $k$-parallelogram polyominoes is readily obtained by summing all the generating functions of the classes $\mathbb{P}_{s}, s \leq k$.

\subsection{Generating function of $k$-parallelogram polyominoes}

The following theorem establishes a criterion for translating the decomposition of Proposition 2 into generating functions.

\section{Theorem 1}

i) A polyomino $P$ belongs to $\mathbb{P}_{2}$ if and only if it is obtained from a polyomino of $\mathbb{P}_{1}$ by adding two new paths $\alpha_{2}$ and $\beta_{2}$, which cannot be both empty, where the height of $\alpha_{2}$ is equal to the height of $\beta_{1}$ minus one, and the width of $\beta_{2}$ is equal to the width of $\alpha_{1}$ minus one.

ii) A polyomino $P$ belongs to $\mathbb{P}_{k}, k>2$, if and only if it is obtained from a polyomino of $\mathbb{P}_{k-1}$ by adding two new paths $\alpha_{k}$ and $\beta_{k}$, which cannot be both empty, where $\alpha_{k}$ has the same height of $\beta_{k-1}$ and $\beta_{k}$ has the same width of $\alpha_{k-1}$.

The proof of Theorem 1 directly follows from our decomposition in Proposition 2, where the difference between the case $k=2$ and the other cases is clearly explained. We would like to point out that if $P$ belongs to $\overline{\mathbb{P}}_{k}$, then neither $\alpha_{k}$ nor $\beta_{k}$ can be empty or flat. Following the statement of Theorem 1 , to pass from $k \geq 1$ to $k+1$ we need to introduce following generating functions:

i) the generating function of the sequence $\mathcal{A}(P)$. Such a function is denoted by $A_{k}(x, y, z)$ for up, and by $\bar{A}_{k}(x, y, z)$ for flat $k$-parallelogram polyominoes, respectively, and, for each function, $x+z$ keeps track of the dimensions of $\mathcal{A}(P)$, and $z$ keeps track of the width/height of $\theta_{k}$ alternately, according to the parity of $k$. 
ii) the generating function of the sequence $\mathcal{B}(P)$. Such a function is denoted by $B_{k}(x, y, t)$ for up, and by $\bar{B}_{k}(x, y, t)$ for flat $k$-parallelogram polyominoes, respectively, and here $y+t$ keeps track of the dimensions of $\mathcal{B}(P)$, and the variable $t$ keeps track of the height/width of $\bar{\theta}_{k}$ alternately, according to the parity of $k$.

Now the generating functions $G f_{k}^{U}(x, y, z, t), G f_{k}^{R}(x, y, z, t)$ and $\overline{G f}_{k}(x, y, z, t)$, of the classes $\mathbb{P}_{k}^{U}$, $\mathbb{P}_{k}^{R}$, and $\overline{\mathbb{P}}_{k}$, respectively, are clearly obtained as follows:

$$
\begin{aligned}
G f_{k}^{U}(x, y, z, t) & =A_{k}(x, y, z) \cdot B_{k}(x, y, t) \\
\overline{G f}_{k}(x, y, z, t) & =\bar{A}_{k}(x, y, z) \cdot \bar{B}_{k}(x, y, t) \\
G f_{k}(x, y, z, t) & =G f_{k}^{U}(x, y, z, t)+G f_{k}^{R}(y, x, t, z)+\overline{G f}_{k}(x, y, z, t) .
\end{aligned}
$$

Then, setting $z=t=y=x$, we have the generating functions according to the semi-perimeter. Since $G f_{k}^{U}(x, y, z, t)=G f_{k}^{R}(y, x, t, z)$, for all $k$, then starting from now, we will study only the flat and the up classes.

The case $k=0 . \quad$ The class $\mathbb{P}_{0}$ is simply made of horizontal and vertical bars of any length. We keep this case distinct from the others since it is not useful for the inductive step, so we simply use the variables $x$ and $y$, which keep track of the width and the height of the polyomino, respectively. The generating function is trivially equal to

$$
G f_{0}(x, y)=x y+\frac{x^{2} y}{1-x}+\frac{x y^{2}}{1-y},
$$

where the term $x y$ corresponds to the unit cell, and the other terms to the horizontal and vertical bars, respectively.

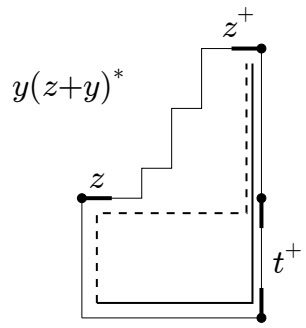

(a)

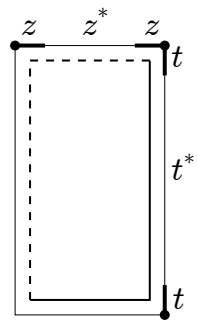

(b)

Figure 5: (a) A polyomino $\in \mathbb{P}_{1}^{U}$ and $(b)$ a polyomino in $\overline{\mathbb{P}}_{1}$.

The case $k=1$. Following our decomposition and Figure 5, we easily obtain

$$
A_{1}(x, y, z)=\frac{z^{2} y}{(1-z-y)(1-z)}, \quad B_{1}(x, y, t)=t+\frac{t^{2}}{1-t} .
$$

We point out that we have written $B_{1}$ as the sum of two terms because, according to Corollary 1 , we have to treat the case when $\beta_{1}$ is made by a north unit step separately from the other cases. To this aim, we set 
$\hat{B}_{1}(x, y, t)=\frac{t^{2}}{1-t}$. Moreover, we have

$$
\bar{A}_{1}(x, y, z)=\frac{z^{2}}{1-z}, \quad \bar{B}_{1}(x, y, t)=\frac{t^{2}}{1-t} .
$$

According to (1) and (2), we have that

$$
G f_{1}^{U}(x, y, z, t)=\frac{t y z^{2}}{(1-t)(1-z)(1-y-z)} \quad \overline{G f}_{1}(x, y, z, t)=\frac{t^{2} z^{2}}{(1-t)(1-z)} .
$$

Now, according to [3], and setting all variables equal to $x$, we have the generating function of 1-parallelogram polyominoes

$$
G f_{1}(x)=\frac{x^{4}(2 x-3)}{(1-x)^{2}(1-2 x)}
$$

The case $k=2$. Now we can use the inductive step, recalling that the computation of the case $k=2$ will be slightly different from the other cases, as explained in Theorem 1 . Using the pictures in Figure 6 we can calculate the generating functions

$$
\begin{gathered}
A_{2}(x, y, z)=z \cdot A_{1}\left(x, y, \frac{x}{1-z}\right)=\frac{x^{2} y z}{(1-x-y-z-y z)(1-x-z)} \\
B_{2}(x, y, t)=\frac{y}{1-t}+t \cdot \hat{B}_{1}\left(x, y, \frac{y}{1-t}\right)=\frac{y-y^{2}}{(1-y-t)}=y+\frac{y t}{1-y-t} \\
\bar{A}_{2}(x, y, z)=z \cdot \bar{A}_{1}\left(x, y, \frac{x}{1-z}\right)=\frac{x^{2} z}{(1-z)(1-x-z)} \\
\bar{B}_{2}(x, y, t)=t \cdot \bar{B}_{1}\left(x, y, \frac{y}{1-t}\right)=\frac{y^{2} t}{(1-t)(1-y-t)} .
\end{gathered}
$$

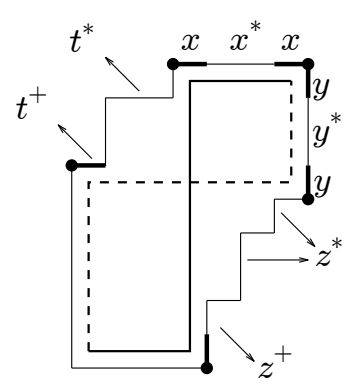

(a)

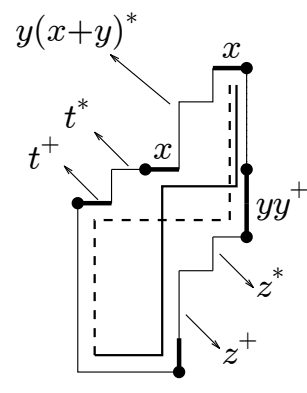

(b)

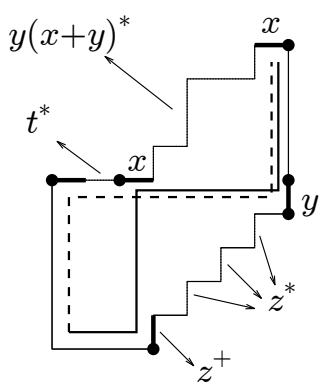

(c)

Figure 6: $(a)$ A polyomino in $\overline{\mathbb{P}}_{2},(b)$ a polyomino in $\mathbb{P}_{2}^{U}$ in which $\beta_{1}$ has at least two north steps and $(c)$ a polyomino in $\mathbb{P}_{2}^{U}$ in which $\beta_{1}$ is equal to an unit north step. 
We observe that the performed substitutions allow us to add the contribution of the terms $\alpha_{2}$ and $\beta_{2}$ from the generating functions obtained for $k=1$. Then, using formulas (1), (2) and (3), and setting all variables equal to $x$, it is straightforward to obtain the generating function according to the semi-perimeter:

$$
G f_{2}(x)=\frac{x^{5}\left(2-5 x+3 x^{2}-x^{3}\right)}{(1-x)^{2}(1-2 x)^{2}\left(1-3 x+x^{2}\right)}
$$

The case $k>2$. The generating functions for the case $k>2$ are obtained in a similar way. Here, for simplicity sake, we set $\hat{B}_{k}(x, y, t)=B_{k}(x, y, t)-y$; this trick will help us treat separately the case when $\beta_{1}$ is made by a north unit step. Then we have

$$
\begin{aligned}
A_{k}(x, y, z) & =\frac{z}{1-z} \cdot A_{k-1}\left(x, y, \frac{x}{1-z}\right) \\
B_{k}(x, y, t) & =\frac{y}{1-t}+\frac{t}{1-t} \cdot \hat{B}_{k-1}\left(x, y, \frac{y}{1-t}\right) \\
\bar{A}_{k}(x, y, z) & =\frac{z}{1-z} \cdot \bar{A}_{k-1}\left(x, y, \frac{x}{1-z}\right) \\
\bar{B}_{k}(x, y, t) & =\frac{t}{1-t} \cdot \bar{B}_{k-1}\left(x, y, \frac{y}{1-t}\right) .
\end{aligned}
$$

We remark that (4), (5), (6) and (7) slightly differ from the respective formulas for $k=2$, according to the statement of Theorem 1 . Using the previous formulas we are now able to obtain an expression for $F_{k}(x)$, for all $k>2$.

\subsection{A formula for the number of $k$-parallelogram polyominoes}

In this section we show a simpler way to express the generating function of $\mathbb{P}_{k}$. First we need to define the following polynomials:

$$
\begin{cases}F_{0}(x, z)=F_{1}(x, z) & =1 \\ F_{2}(x, z) & =1-z \\ F_{k}(x, z) & =F_{n-1}(x, z)-x F_{n-2}(x, z) .\end{cases}
$$

These objects are already known as Fibonacci polynomials [6].

In the sequel, unless otherwise specified, we will denote $F_{k}(x, x)$ with $F_{k}$. The closed Formula of $F_{k}$ is:

$$
F_{k}=\frac{b(x)^{k+1}-a(x)^{k+1}}{\sqrt{1-4 x}} .
$$

where $a(x)$ and $b(x)$ are the solutions of the equation $X^{2}-X+x=0, a(x)=\left(\frac{1-\sqrt{1-4 x}}{2}\right)$ and $b(x)=\left(\frac{1+\sqrt{1-4 x}}{2}\right)$.

Our aim is to express the functions $A_{k}, B_{k}, \bar{A}_{k}$, and $\bar{B}_{k}$ in terms of the previous polynomials. In order to do this we need to prove the following lemma:

Lemma 1 For every $k$

$$
F_{k}\left(x, \frac{x}{1-z}\right)=\frac{F_{k+1}(x, z)}{1-z} .
$$


The proof is easily obtained by induction. Placing $y=x$, we can write $A_{1}(x, z)=\frac{x z^{2}}{F_{2}(x, z) F_{3}(x, z)}$ and iterating (4), and using Lemma 1, we obtain

$$
A_{k}(x, z)=\frac{z x^{k+1}}{F_{k+1}(x, z) F_{k+2}(x, z)} .
$$

Performing the same calculations on the other functions we obtain:

$$
\begin{aligned}
B_{k}(x, z) & =\frac{x F_{k}}{F_{k+1}(x, z)} \\
\bar{A}_{k}(x, z) & =\bar{B}_{k}(x, z)=\frac{z x^{k}}{F_{k}(x, z) \cdot F_{k+1}(x, z)}
\end{aligned}
$$

From these new functions, by setting all variables equal to $x$, we can calculate the generating function of the class $\mathbb{P}_{k}$ in an easier way:

$$
G f_{k}(x)=2 A_{k}(x, x) B_{k}(x, x)+\left(\bar{A}_{k}\right)^{2}(x, x) .
$$

Then we have the following:

Theorem 2 The generating function of the whole class of k-parallelogram polyominoes is given by

$$
P_{k}(x)=\sum_{n=0}^{k} G f_{n}(x)=x^{2} \cdot\left(\frac{F_{k+1}}{F_{k+2}}\right)^{2}-x^{2} \cdot\left(\frac{F_{k+1}}{F_{k+2}}-\frac{F_{k}}{F_{k+1}}\right)^{2} .
$$

As an example, the generating functions of $\mathbb{P}_{k}$ for the first values of $k$ are:

$$
\begin{array}{ll}
P_{0}(x)=\frac{x^{2}(1+x)}{1-x} & P_{1}(x)=\frac{x^{2}\left(1-2 x+2 x^{2}\right)}{(-1+x)^{2}(1-2 x)} \\
P_{2}(x)=\frac{x^{2}(1-x)\left(1-4 x+4 x^{2}+x^{3}\right)}{(1-2 x)^{2}\left(1-3 x+x^{2}\right)} & P_{3}(x)=\frac{x^{2}(1-2 x)\left(1-6 x+11 x^{2}-6 x^{3}+2 x^{4}\right)}{(1-x)(1-3 x)\left(1-3 x+x^{2}\right)^{2}}
\end{array}
$$

In [6] it is proved that $x F_{k} / F_{k+1}$ is the generating function of plane trees having height less than or equal to $k+1$. Hence, $P_{k}(x)$ is the difference between the generating functions of pairs of trees having height at most $k+3$, and pairs of trees having height exactly equal to $k+2$. It would be interesting to provide a combinatorial explanation to this fact.

As one would expect we have the following corollary:

Corollary 2 Let $C(x)=\frac{1-\sqrt{1-4 x}}{2 x}$ be the generating function of Catalan numbers, we have:

$$
\lim _{k \rightarrow \infty} P_{k}(x)=C(x)
$$

Proof: We have that $C(x)$ satisfies the equation $C(x)=1+x C^{2}(x)$, and $a(x) b(x)=x, a(x)=x C(x)$, so we can write

$$
F_{k}=\frac{1-x^{k+1} C^{2(k+1)}(x)}{C^{k+1} \sqrt{1-4 x}}
$$


Now we can prove the following statements:

$$
\lim _{k \rightarrow \infty} \frac{F_{k}}{F_{k+1}}=C(x), \quad \lim _{k \rightarrow \infty}\left(\frac{F_{k}}{F_{k+1}}\right)^{2}=\frac{C(x)-1}{x}, \quad \lim _{k \rightarrow \infty} \frac{F_{k}}{F_{k+2}}=\frac{C(x)-1}{x} .
$$

From Theorem 2, and using the above results, we obtain the desired proof.

\section{References}

[1] M. Bousquet-Mèlou, A method for the enumeration of various classes of column-convex polygons, Disc. Math. 154 (1996) 1-25.

[2] G. Castiglione, A. Frosini, A. Restivo, S. Rinaldi, A tomographical characterization of $L$-convex polyominoes, Proc. of Discrete Geometry for Computer Imagery 12th International Conference (DGCI 2005), Poitiers, France, April 11-13, (2005), E. Andres, G. Damiand, P. Lienhardt (Eds.), Lecture Notes in Computer Science, vol. 3429, (2005), pp. 115-125.

[3] G. Castiglione, A. Frosini, E. Munarini, A. Restivo, S. Rinaldi, Combinatorial aspects of L-convex polyominoes, European J. Combin. 28 (2007) 1724-1741.

[4] G. Castiglione, A. Frosini, A. Restivo, S. Rinaldi, Enumeration of $L$-convex polyominoes by rows and columns, Theor. Comput. Sci., vol. 347, (2005), pp. 336-352.

[5] G. Castiglione, A. Restivo, Ordering and Convex Polyominoes, in: M. Margenstern (Ed.), Machines, Computations, and Universality, MCU 2004, Saint Petersburg, Russia, September 21-24, (2004), in: Revised Selected Papers: Lecture Notes in Computer Science, vol. 3354, Springer, (2005).

[6] N.G. De Bruijn, D.E. Knuth, S.O. Rice, The average height of planted plane trees, In Graph Theory and Computing (R.C. Read, ed.), Academic Press (1972) 15-22.

[7] M. Delest, X. Viennot, Algebraic languages and polyominoes enumeration, Theor. Comp. Sci., vol. 34, (1984),pp. 169-206.

[8] E. Duchi, S. Rinaldi, G. Schaeffer, The number of Z-convex polyominoes, it Advances in Applied Math., vol. 40, (2008), pp. 54-72.

[9] A. Micheli, D. Rossin, Asymptotics of $k$-convex, (personal comunication).

[10] OEIS Foundation Inc. (2011), The On-Line Encyclopedia of Integer Sequences, http:// oeis. org

[11] R.P. Stanley, Enumerative Combinatorics, Vol. 2, Cambridge University Press, Cambridge (1999). 